JOURNAL OF

FUNCTION SPACES AND APPLICATIONS

Volume 3, Number 2 (2005), 183-208 (c) 2005, Scientific Horizon http://www.jfsa.net

\title{
Sobolev type inequalities in ultrasymmetric spaces with applications to Orlicz-Sobolev embeddings
}

\author{
Evgeniy Pustylnik \\ (Communicated by Lech Maligranda)
}

2000 Mathematics Subject Classification. 46E30, 46E35.

Keywords and phrases. Sobolev embeddings, rearrangement-invariant spaces, Orlicz spaces.

Abstract. Let $D^{k} f$ mean the vector composed by all partial derivatives of order $k$ of a function $f(x), x \in \Omega \subset \mathbb{R}^{n}$. Given a Banach function space $A$, we look for a possibly small space $B$ such that $\|f\|_{B} \leq c\left\|\left|D^{k} f\right|\right\|_{A}$ for all $f \in C_{0}^{k}(\Omega)$. The estimates obtained are applied to ultrasymmetric spaces $A=L_{\varphi, E}, B=L_{\psi, E}$, giving some optimal (or rather sharp) relations between parameter-functions $\varphi(t)$ and $\psi(t)$ and new results for embeddings of OrliczSobolev spaces.

\section{Introduction}

Let $W_{p}^{k}=W_{p}^{k}(\Omega)$ mean a standard Sobolev space on the bounded open set $\Omega \subset \mathbb{R}^{n}$ (for simplicity, we may assume $|\Omega|=1$ ). As known, the classical Sobolev embedding

$$
W_{p}^{k} \hookrightarrow L_{q}, \quad 1<p<n / k, \quad q=n p /(n-k p)
$$


(when it is true) gives the best possible value of the exponent $q$ for a given $p$, but the corresponding space $L_{q}$ is not optimal among all rearrangement invariant spaces suitable for such an embedding. The best possible result was obtained by O'Neil [21] and Dikarev [9], using the Lorentz spaces

$$
W_{p}^{k} \hookrightarrow L(q, p), \quad 1<p<n / k, \quad q=n p /(n-k p)
$$

(recall that $L(q, p)$ is essentially smaller than $L_{q}$, since $q>p$ ). The situation is still more complicated for the "limiting" case $p=n / k$, when the embeddings (1.1) and (1.2) are true for any $q<\infty$ but not for $q=\infty$ that should follow from the definition $q=n p /(n-k p)$. This case was studied in 60's by Yudovich [32], Pokhozhaev [24], Trudinger [30], Peetre [22] etc. with the help of Orlicz spaces

$$
W_{n / k}^{k} \hookrightarrow L_{\Phi}, \quad \Phi(u)=e^{u^{\lambda}}, \quad \lambda=\frac{n}{n-k} .
$$

Although the exponent $\lambda=n /(n-k)$ was later shown as best possible, the space $L_{\Phi}$ itself turned out to be not such and was replaced in 1979-1980 by essentially smaller (and, in fact, the smallest possible) rearrangementinvariant space $B$ with the norm

$$
\|f\|_{B}=\left\|t^{-k / n}\left(\ln \frac{e}{t}\right)^{-1} f^{*}(t)\right\|_{L_{n / k}(0, \infty)},
$$

where, as usual, $f^{*}$ stands for the non-increasing rearrangement of the function $f$ (see, e.g., Maz'ya [18], Hansson [12], Brezis-Wainger [4]).

Involving various functional spaces as the range of embedding, it is natural to use such spaces also in definition of Sobolev spaces themselves. The main idea of their classical definition is to put certain summability conditions not only on considered functions, but also on their derivatives up to some fixed order. These conditions have usually a form of belonging to corresponding $L_{p}$-spaces. Thus the direct way for generalization is replacement of $L_{p}$ by other function spaces. The mostly used and studied substitutes for this are Lorentz and Orlicz spaces, but the last time some papers began to consider more general spaces (see, e.g., [7], [10] etc.).

Let $A(\Omega)$ be a Banach function space on some open set $\Omega \subset \mathbb{R}^{n}$. For a given positive integer $k$, a (generalized) Sobolev space $W_{A}^{k}(\Omega)$ is defined as the collection of all functions $f \in A(\Omega)$ such that all their weak (distributional) derivatives up to order $k$ also belong to $A(\Omega)$. This space 
is usually normed by

$$
\|f\|_{W_{A}^{k}(\Omega)}=\sum_{|\alpha|=0}^{k}\left\|\frac{\partial^{|\alpha|} f}{\partial^{\alpha_{1}} x_{1} \cdots \partial^{\alpha_{n}} x_{n}}\right\|_{A}, \quad|\alpha|=\alpha_{1}+\cdots+\alpha_{n} .
$$

The main problem is to find a space $B(\Omega)$ (as small as possible), for which $W_{A}^{k}(\Omega) \hookrightarrow B(\Omega)$.

For the normed and quasinormed rearrangement-invariant (r.i.) spaces $A, B$, this problem is discussed rather deeply and in detail in the paper [10] (with supplements in [23]), proving some general criteria of optimality of the spaces involved. Moreover, in the case of a given range space $B$, the opposite problem is solved there explicitly, with analytical formula for the corresponding optimal domain space $A$. Unfortunately, the initial problem of optimal range space $B$ for a given $A$ is much more complicated, and the corresponding general solution in the above mentioned papers is not explicit enough, containing some actions hard for computations. Therefore it makes sense to find other possible estimates for range spaces $B$, if even they are not optimal but sharp and have a workable analytical form.

Another way for studying Sobolev type embeddings between r.i. spaces is proposed in [20], containing a rather sharp inequality for the norm (1.3) of a new type. In fact, this inequality gives an embedding of $W_{A}^{k}$ into some set of functions $Y_{k}(A)$ which even may be not a linear space. In the present paper we show that this inequality remains true also for some non r.i. spaces $A$. Basing on such inequalities, we are able to obtain optimal (or rather sharp) estimates for the range spaces $B$ as above with explicit analytical description. This way turns out to be particularly effective for the ultrasymmetric spaces, defined in [27]. Note that this class of spaces comprises many classical r.i. spaces such as Lorentz, Lorentz-Zygmund and so on. And in the very difficult "limiting" case of embeddings (see Section 6 below), this class includes most of Orlicz spaces, that leads to rather sharp estimations for Orlicz-Sobolev norms.

As in the case of classical spaces (see, e.g., [19], [29]), under rather mild conditions on $A(\Omega)$, the norm (1.3) is equivalent to the sum containing only derivatives of the zero and of the highest order. Comparison of the norms $\|f\|_{B}$ and $\|f\|_{A}$ (without derivatives) is a usual part of the function space theory, hence a new task is to compare $\|f\|_{B}$ with the sum of norms in $A$ of the highest derivatives; the last, in turn, is equivalent to $\left\|\left|D^{k} f\right|\right\|_{A}$, where $D^{k}$ stands for the vector composed by all derivatives of order $k$. The corresponding inequalities are said to be of Sobolev type; in the present paper we will prove such inequalities for u.s. spaces with consequences for Orlicz spaces. 
It should be noticed that during the last years the Orlicz-Sobolev embeddings were studied rather intensively in a number of papers by A. Cianchi (see, e.g., [5] and the references there). Moreover, the paper [5] gives some formulas for optimal r.i. embeddings of spaces $W_{A}^{1}(\Omega)$ with arbitrary Orlicz spaces $A$. In the present paper we consider Orlicz-Sobolev spaces of any order $k \leq n-1$, but only in the context of ultrasymmetric spaces. Unfortunately, we cannot compare our results from Section 6 (except for the formula (6.2)) with results from [5] even when $k=1$, since the general formulas in [5], Theorem 1.1, are rather hard for concrete calculations.

Throughout the paper we do not differentiate spaces with equivalent norms and quasi-norms. The letters $C, c, c_{1}$ etc. will stand for any nonspecified constant and the records like $f \approx g$ will mean equivalence of considered functions (expressions). Given a function set (space) $X(\Omega)$, we denote by $X_{0}(\Omega)$ the subset of functions from $X(\Omega)$ having compact supports in $\Omega$.

\section{Preliminaries}

From now on let $\Omega$ be an open, maybe unbounded, set in $\mathbb{R}^{n}$ with the standard Lebesgue measure. A Banach space $A$ of measurable functions $f: \Omega \mapsto \mathbb{R}$ is called function space if, for any $g \in A(\Omega)$ and for any measurable $f$ such that $|f(x)| \leq|g(x)|$ for a.e. $x \in \Omega$, it follows that $f \in A(\Omega)$ and $\|f\|_{A(\Omega)} \leq\|g\|_{A(\Omega)}$. Let, as usual,

$$
f^{*}(t)=\inf \{\lambda>0: \operatorname{mes}\{x \in \Omega:|f(x)|>\lambda\} \leq t\}, \quad t>0 .
$$

The function space $A(\Omega)$ is called rearrangement-invariant (r.i.) if, for any $g \in A(\Omega)$, it contains all functions $f$ such that $f^{*}(t)=g^{*}(t)$ for all $t>0$ and $\|f\|_{A(\Omega)}=\|g\|_{A(\Omega)}$. Let us assume that every $f(x)=0$ whenever $x \in \mathbb{R}^{n} \backslash \Omega$, then any r.i. space $A(\Omega)$ can be "reduced" to one-dimensional case $A=A(0, \infty)$ consisting of all $g:(0, \infty) \mapsto \mathbb{R}$ such that $g^{*}(t)=f^{*}(t)$ for some function $f \in A(\Omega)$.

Any r.i. space is characterized by the fundamental function $\varphi_{A}(s)=$ $\left\|\chi_{(0, s)}(t)\right\|_{A}$ and their extension indices

$$
\pi_{\varphi}=\lim _{s \rightarrow 0} \frac{\ln m_{\varphi}(s)}{\ln s}, \quad \rho_{\varphi}=\lim _{s \rightarrow \infty} \frac{\ln m_{\varphi}(s)}{\ln s}, \quad m_{\varphi}(s)=\sup _{t} \frac{\varphi_{A}(t s)}{\varphi_{A}(t)} .
$$

Another characteristic is the dilation function

$$
d_{A}(s)=\sup _{f \in A} \frac{\|f(t / s)\|_{A}}{\|f(t)\|_{A}}
$$


which is used for definition of the so-called Boyd indices

$$
\pi_{A}=\lim _{s \rightarrow 0} \frac{\ln d_{A}(s)}{\ln s}, \quad \rho_{A}=\lim _{s \rightarrow \infty} \frac{\ln d_{A}(s)}{\ln s} .
$$

In general $0 \leq \pi_{\varphi} \leq \pi_{A} \leq \rho_{A} \leq \rho_{\varphi}$, but for many important spaces ( $L_{p}$, Orlicz, Lorentz etc.) $\pi_{A}=\pi_{\varphi}$ and $\rho_{A}=\rho_{\varphi}$.

Remark. The dilation function may be defined for nonrearrangementinvariant spaces too, but in this case it can be infinite for some values of $s$.

For the main definitions and properties concerning Banach function and rearrangement-invariant spaces as well as interpolation theory, we refer the reader to the monograph [3]. It is assumed there additionally that all considered spaces have the Fatou property so that any Banach r.i. space $E$ with this property is exact interpolation between $L_{1}$ and $L_{\infty}$. In other words, any such a space can be defined as $E=\mathcal{F}\left(L_{1}, L_{\infty}\right)$ for some interpolation functor $\mathcal{F}$. Given a Banach r.i. space $E$, we apply the same functor for defining another space $\widetilde{E}=\mathcal{F}\left(\widetilde{L}_{1}, \widetilde{L}_{\infty}\right)$, where

$$
\|f\|_{\widetilde{L}_{1}}=\int_{0}^{\infty}|f(t)| \frac{d t}{t}, \quad\|f\|_{\widetilde{L}_{\infty}}=\|f\|_{L_{\infty}} .
$$

Except $\widetilde{L}_{\infty}$, every $\widetilde{E}$ is not a usual r.i. space but rearrangement-invariant with respect to the measure $d t / t$. These spaces will be used for the following definition (see [27]):

Definition. A rearrangement-invariant space $G$ is called ultrasymmetric (u.s.) if its norm is equivalent to $\left\|\varphi(t) f^{*}(t)\right\|_{\widetilde{E}}$ for some positive increasing parameter-function $\varphi(t)$ with finite extension indices and some parameterspace $\widetilde{E}$. For such spaces, we will use a notation $G=L_{\varphi, E}$.

As shown in [27], an r.i. space $G$ with $\pi_{G}>0$ is u.s. if and only if it is interpolation between corresponding Lorentz space $\Lambda_{\varphi}$ and Marcinkiewicz space $M_{\varphi}$ with the norms

$$
\|f\|_{\Lambda_{\varphi}}=\int_{0}^{\infty} f^{*}(t) d \varphi(t), \quad\|f\|_{M_{\varphi}}=\sup _{t} f^{*}(t) \varphi(t),
$$

thus the fundamental function $\varphi_{G}(t)$ is equivalent to $\varphi(t)$. Some other properties of u.s. spaces, proved in [27], also will be used below.

We will also need the following Hardy operators

$$
P f(t)=\frac{1}{t} \int_{0}^{t} f(s) d s, \quad Q f(t)=\int_{t}^{\infty} f(s) \frac{d s}{s}
$$


and the notation $f^{* *}(t)=P\left(f^{*}\right)(t)$. We say the space $A(\Omega)$ has $(P)$ (or $(Q))$ property if the corresponding Hardy operator is bounded on $A(0, \infty)$. For r.i. spaces, the $(P)$ property is equivalent to the inequality $\rho_{A}<1$ and the $(Q)$ property is equivalent to the inequality $\pi_{A}>0$.

Let $0 \leq k<n$. We say the space $A$ has $Q(k)$ property if

$$
Q(k, A)=\int_{1}^{\infty} s^{k / n} d_{A}\left(\frac{1}{s}\right) \frac{d s}{s}<\infty
$$

(it is easy to see that the $Q(0)$ property coincides with $(Q)$ ). In the case of a r.i. space $A$, this property is equivalent to the inequality $\pi_{A}>k / n$. If a Banach function space $A$ has $Q(k)$ property then

$$
\left\|t^{-k / n} Q f(t)\right\|_{A} \leq Q(k, A)\left\|t^{-k / n} f(t)\right\|_{A} .
$$

Indeed, using the generalized Minkowski's inequality (see, e.g., [14], p. 45), we obtain that

$$
\begin{aligned}
\left\|t^{-k / n} Q f(t)\right\|_{A} & =\left\|\int_{1}^{\infty} t^{-k / n} f(s t) \frac{d s}{s}\right\|_{A} \\
& \leq \int_{1}^{\infty}\left\|(s t)^{-k / n} f(s t)\right\|_{A} s^{k / n-1} d s \\
& \leq \int_{1}^{\infty} d_{A}\left(\frac{1}{s}\right) s^{k / n-1} d s\left\|t^{-k / n} f(t)\right\|_{A} .
\end{aligned}
$$

Analogously we can show that

$$
\left\|t^{-k / n} P f(t)\right\|_{A} \leq P(k, A)\left\|t^{-k / n} f(t)\right\|_{A}
$$

with

$$
P(k, A)=\int_{0}^{1} s^{k / n} d_{A}\left(\frac{1}{s}\right) d s .
$$

Note that this quantity is finite for any Banach r.i. space $A$, because in this case $d_{A}(s) \leq \max \{1, s\}$. That is why we do not introduce the $P(k)$ property here.

Being decreasing functions, both $f^{*}(t)$ and $f^{* *}(t)$ have limits on infinity; it is easy to see that these limits are equal. Thus $f^{* *}(\infty)=0$ whenever $f \in A \not \supset L_{\infty}(0, \infty)$, and the last property of the space $A$ is provided by any of $Q$ or $Q(k)$ properties.

Any function space $A$ generates an r.i. space $A^{\star}$ with the norm $\|f\|_{A \star}=$ $\left\|f^{* *}\right\|_{A}$ that will be useful in the future discussions. It is easy to check that $d_{A} \star(s) \leq d_{A}(s)$, thus any of $P, Q, Q(k)$ properties extends from $A$ to $A^{\star}$. 


\section{Basic inequalities}

In the last years it was revealed that various first order differential properties of measurable functions $f(x)$ can be characterized, using some special differences $f^{*}(\varepsilon t)-f^{*}(t), 0<\varepsilon<1$, and $f^{* *}(t)-f^{*}(t)$. The first of them was widely studied and used by Kolyada (see, e.g., [13]), Sagher and Shvartsman (see, e.g., [28]), Malý and Pick [17] and some others. The second difference appeared first in a paper by Ulyanov [31], then in the BennettDeVore-Sharpley's characterization of BMO-spaces (see [3], Section 5.7); its behaviour in weighted $L_{p}$-spaces was investigated in [15]. An important role of this difference for new properties of the Sobolev space $W_{p}^{1}$ was stated in the recent paper by Bastero-Milman-Ruiz [2].

Both differences are tightly connected one with another by the relations

$$
\begin{gathered}
f^{*}(\varepsilon t)-f^{*}(t) \leq \frac{1}{\varepsilon}\left(f^{* *}(t)-f^{*}(t)\right), \\
f^{* *}(t)-f^{*}(t) \leq \frac{\varepsilon}{1-\varepsilon} P\left[f^{*}(\varepsilon s)-f^{*}(s)\right](t)+\left(f^{*}(\varepsilon t)-f^{*}(t)\right) .
\end{gathered}
$$

The first of them follows immediately, since

$$
\begin{aligned}
f^{* *}(t) & =\frac{1}{t} \int_{0}^{\varepsilon t} f^{*}(s) d s+\frac{1}{t} \int_{\varepsilon t}^{t} f^{*}(s) d s \\
& \geq \varepsilon f^{*}(\varepsilon t)+(1-\varepsilon) f^{*}(t) .
\end{aligned}
$$

The proof of (3.2) is a bit longer:

$$
\begin{aligned}
f^{* *}(t) & =\frac{1}{t} \int_{0}^{\varepsilon t} f^{*}(s) d s+\frac{1}{t} \int_{\varepsilon t}^{1} f^{*}(s) d s \\
& \leq \frac{\varepsilon}{t} \int_{0}^{t} f^{*}(\varepsilon s) d s+(1-\varepsilon) f^{*}(\varepsilon t),
\end{aligned}
$$

whence

$$
\frac{1}{\varepsilon} f^{* *}(t) \leq \frac{1}{t} \int_{0}^{t}\left[f^{*}(\varepsilon s)-f^{*}(s)\right] d s+f^{* *}(t)+\frac{1-\varepsilon}{\varepsilon} f^{*}(\varepsilon t)
$$

and thus

$$
f^{* *}(t) \leq \frac{\varepsilon}{1-\varepsilon} \cdot \frac{1}{t} \int_{0}^{t}\left[f^{*}(\varepsilon s)-f^{*}(s)\right] d s+f^{*}(\varepsilon t) .
$$

As a consequence, both differences have equivalent norms in any space with the $(P)$ property. 
The use of the difference $f^{*}(\varepsilon t)-f^{*}(t)$ for studying the first derivatives of a function $f(x) \in C^{1}\left(\mathbb{R}^{n}\right)$ with $f^{*}(\infty)=0$ is based on the following fundamental inequality

$$
f^{*}(\varepsilon t)-f^{*}(t) \leq c t^{1 / n}(\nabla f)^{* *}(t),
$$

where $\nabla$ stands for the usual gradient

$$
\nabla f=\left(\frac{\partial f}{\partial x_{1}}, \ldots, \frac{\partial f}{\partial x_{n}}\right)
$$

and $(\nabla f)^{* *}$ means $|\nabla f|^{* *}$. This inequality (in a slightly different form) was proved by Kolyada (see [13]), using the classical Loomis -Whitney theorem. Applying the Hardy operator $P$ to both sides of (3.3) and changing the integration order in the right-hand side, we obtain that also

$$
\frac{1}{t} \int_{0}^{t}\left(f^{*}(\varepsilon s)-f^{*}(s)\right) d s \leq c t^{1 / n}(\nabla f)^{* *}(t) .
$$

Together with the relation (3.2) this gives that

$$
f^{* *}(t)-f^{*}(t) \leq c t^{1 / n}(\nabla f)^{* *}(t),
$$

and this will be the starting point of our discussion in the present paper.

Remark. Another (direct) proof of the inequality (3.4) is given in the paper [2]: first for the spherically symmetric functions $f(x)=g(|x|)$ and then using the Polya-Szegö symmetrization principle in the form proposed by Fournier [11].

For any function space $A$, we obtain from (3.4) immediately that

$$
\left\|t^{-1 / n}\left(f^{* *}(t)-f^{*}(t)\right)\right\|_{A} \leq c\left\|(\nabla f)^{* *}\right\|_{A}
$$

(the result from [2] for the case of $A=L_{n}$ and from [20] for arbitrary Banach r.i. space $A$ ). In contrast to the inequality (3.4), the last inequality can be generalized to higher order derivatives of $f$, using the ideas of [20], namely, that

$$
f^{* *}(t)=Q\left(f^{* *}-f^{*}\right)(t) \quad \text { for } f^{* *}(\infty)=0 .
$$

For instance, if the space $A$ has $Q(1)$ property, then by (2.2), (3.5) and (3.6) we obtain that

$$
\left\|t^{-1 / n} f^{* *}(t)\right\|_{A} \leq c_{1}\left\|(\nabla f)^{* *}(t)\right\|_{A}
$$


and, taking $|\nabla f|$ in place of $f$, we come by (3.4) to the inequality

$$
\left\|t^{-2 / n}\left(f^{* *}(t)-f^{*}(t)\right)\right\|_{A} \leq c\left\|t^{-1 / n}(\nabla f)^{* *}(t)\right\|_{A} \leq c_{2}\left\|\left(D^{2} f\right)^{* *}(t)\right\|_{A}
$$

for any $f \in C^{2}\left(\mathbb{R}^{n}\right)$ with $f^{* *}(\infty)=(\nabla f)^{* *}(\infty)=0$. Here $D^{2}$ stands for the vector composed by all second derivatives and $\left(D^{2} f\right)^{* *}$ means $\left|D^{2} f\right|^{* *}$.

Proceeding inductively, we come to the following main assertion.

Theorem 3.1. Let $A(\Omega)$ be a Banach function space with some open set $\Omega \subset \mathbb{R}^{n}$. Let $k$ be an arbitrary integer such that $1 \leq k \leq n-1$. If $A$ has $Q(k-1)$ property and a function $f \in C^{k}\left(\mathbb{R}^{n}\right)$ is such that $f^{* *}(\infty)=(\nabla f)^{* *}(\infty)=\ldots=\left(D^{k-1} f\right)^{* *}(\infty)=0$, then

$$
\left\|t^{-k / n}\left(f^{* *}(t)-f^{*}(t)\right)\right\|_{A} \leq c\left\|\left(D^{k} f\right)^{* *}(t)\right\|_{A} .
$$

(in the same style as before, $\left(D^{k} f\right)^{* *}$ means $\left|D^{k} f\right|^{* *}$ for the vector $D^{k}$ composed by all derivatives of order $k$ ).

Proof. Assume that (3.7) is already proved for $k-1$ :

$$
\left\|t^{-(k-1) / n}\left(f^{* *}(t)-f^{*}(t)\right)\right\|_{A} \leq c\left\|\left(D^{k-1} f\right)^{* *}(t)\right\|_{A} .
$$

Due to $Q(k-1)$ property and $(2.2)$, this implies that

$$
\left\|t^{-(k-1) / n} f^{* *}(t)\right\|_{A} \leq c\left\|\left(D^{k-1} f\right)^{* *}(t)\right\|_{A}
$$

for any $f(x) \in C^{k-1}\left(\mathbb{R}^{n}\right)$ with $f^{* *}(\infty)=\ldots=\left(D^{k-2} f\right)^{* *}(\infty)=0$ and thus for any derivative $f_{i}=\partial f / \partial x_{i}, i=1, \ldots, n$ in place of $f(x)$. Since $\left|D^{k-1} f_{i}\right| \leq\left|D^{k} f\right|$, we obtain that

$$
\left\|t^{-(k-1) / n} f_{i}^{* *}(t)\right\|_{A} \leq c\left\|\left(D^{k} f\right)^{* *}(t)\right\|_{A}
$$

Summing such inequalities over all $i=1, \ldots, n$ and using properties of the "double-star" operation

$$
(\nabla f)^{* *} \leq\left(\left|f_{1}\right|+\cdots+\left|f_{n}\right|\right)^{* *} \leq f_{1}^{* *}+\cdots+f_{n}^{* *},
$$

we arrive at inequality

$$
\left\|t^{-(k-1) / n}(\nabla f)^{* *}(t)\right\|_{A} \leq c\left\|\left(D^{k} f\right)^{* *}(t)\right\|_{A},
$$

which by (3.4) leads to (3.7).

Note that all requirements to functions $f(x)$ from this theorem are fulfilled if we consider only $f \in C_{0}^{k}(\Omega)$. 


\section{Some consequences for rearrangement-invariant spaces}

If $A$ is an r.i. space with $(P)$ property then we may omit two stars in the right-hand term of the inequality (3.7). And if we consider only functions $f \in C_{0}^{k}(\Omega)$ or if the boundary $\partial \Omega$ satisfies some minimal smoothness conditions sufficient for the extension theorems (see, e.g., [29]), then we may replace $D^{k} f$ by various reduced expression, for example, by $k$-th order gradient (see [1])

$$
\nabla^{k} f=\left\{\begin{array}{cl}
\Delta^{k / 2} f & \text { for even } k \\
\nabla\left(\Delta^{(k-1) / 2}\right) f & \text { for odd } k,
\end{array}\right.
$$

where $\Delta$ means the standard Laplace operator. We arrive at the inequality

$$
\left\|t^{-k / n}\left(f^{* *}(t)-f^{*}(t)\right)\right\|_{A} \leq c\left\|\left|\nabla^{k} f\right|\right\|_{A},
$$

which in such a form was obtained in [20].

Remark. The right-hand term of the inequality (3.7) is the norm of $\left|D^{k} f\right|$ in the space $A^{\star}$ which is always rearrangement-invariant. Thus if even $A$ is arbitrary function space with $(P)$ property, the inequality (3.7) for any function $f \in C_{0}^{k}(\Omega)$ is equivalent to an analog of inequality (4.1), namely, to

$$
\left\|t^{-k / n}\left(f^{* *}(t)-f^{*}(t)\right)\right\|_{A} \leq c\left\|\left(\nabla^{k} f\right)^{*}\right\|_{A},
$$

In the case of r.i. spaces the result of Theorem 3.1 is optimal in the following sense.

Theorem 4.1. Let, under conditions of Theorem 3.1, A be a Banach r.i. space with $(P)$ property and let another Banach r.i. space $B$ be such that

$$
\|f\|_{B} \leq c_{1}\left\|\left|D^{k} f\right|\right\|_{A}
$$

for all admissible $f$. Then

$$
\left\|f^{* *}\right\|_{B} \leq c_{2}\left\|t^{-k / n}\left(f^{* *}(t)-f^{*}(t)\right)\right\|_{A} .
$$

Proof. We shall use the following result from [10], Section 3. For any nonnegative continuous function $g(t), t \in(0,|\Omega|)$, there exists a function $u \in C^{k}(\Omega)$ such that $\left|\nabla^{k} u(x)\right|=g\left(|x|^{n}\right)$ for all $x \in \Omega$ and 


$$
u^{*}(\varepsilon t) \geq c \int_{t}^{|\Omega|} g(s) s^{k / n-1} d s
$$

with some $\varepsilon$ independent of $g$. Combining this result with the inequality (4.3), we obtain that the linear operator

$$
\left(S_{k} g\right)(t)=\int_{t}^{|\Omega|} s^{k / n} g(s) \frac{d s}{s}
$$

is bounded from $A$ to $B$, which, for the function $g(s)=s^{-k / n}\left(f^{* *}(s)-\right.$ $\left.f^{*}(s)\right)$, gives

$$
\left\|\int_{t}^{|\Omega|}\left(f^{* *}(s)-f^{*}(s)\right) \frac{d s}{s}\right\|_{B} \leq c\left\|t^{-k / n}\left(f^{* *}(t)-f^{*}(t)\right)\right\|_{A} .
$$

But $\left(f^{* *}(t)-f^{*}(t)\right) / t=-\left(f^{* *}(t)\right)^{\prime}$, thus the last inequality takes a form

$$
\left\|f^{* *}(t)-f^{* *}(|\Omega|)\right\|_{B} \leq c\left\|t^{-k / n}\left(f^{* *}(t)-f^{*}(t)\right)\right\|_{A} .
$$

If $|\Omega|=\infty$ this finishes the proof, since $f^{* *}(\infty)=0$. Alternatively, $\left\|f^{* *}(|\Omega|)\right\|_{B}=\|f\|_{L_{1}}\left\|\chi_{(0,|\Omega|)}\right\|_{B}$ and again $f^{* *}(|\Omega|)$ may be omitted.

Corollary. If $A$ is a r.i. space such that $\rho_{A}<1$ and $\pi_{A}>k / n$, then

$$
\|f\|_{X}=\left\|t^{-k / n} f^{* *}(t)\right\|_{A} \leq c\left\|\left|D^{k} f\right|\right\|_{A} \quad \text { for all } f \in C_{0}^{k}(\Omega),
$$

and $X$ is the smallest r.i. space providing such an inequality.

Proof. Indeed, by (3.6) and (2.2) we obtain that

$$
\left\|t^{-k / n} f^{* *}(t)\right\|_{A}=\left\|t^{-k / n} Q\left(f^{* *}-f^{*}\right)(t)\right\|_{A} \leq Q(k, A)\left\|t^{-k / n}\left(f^{* *}(t)-f^{*}(t)\right)\right\|_{A}
$$

and may use the inequality (3.7). Optimality of the space $X$ follows then immediately from Theorem 4.1.

The last assertion gives, by the way, that in the case, when the space $A$ has $Q(k)$ property, the set of function

$$
Y_{k}(A)=\left\{f: t^{-k / n}\left(f^{* *}(t)-f^{*}(t)\right) \in A\right\}
$$

is a linear space coinciding with r.i. space $X$. If $A$ does not possess this property, the set $Y_{k}(A)$ may be not linear at all, and the problem on optimal r.i. space $B$ such that $\|f\|_{B} \leq c\left\|\left|D^{k} f\right|\right\|_{A}$ turns out to be very difficult. Nevertheless, even in such cases, Theorem 3.1 allows us to construct suitable 
spaces $B$ which are rather "close" to optimality. For this purpose, we can use the following statement.

Theorem 4.2. Let a function space $A$ be as in Theorem 3.1 and let another function space $B$ be such that the operator (4.5) is bounded from $A$ to $B$. Then

$$
\left\|f^{* *}\right\|_{B} \leq c\left(\left\|\left(D^{k} f\right)^{* *}\right\|_{A}+\|f\|_{L_{1}}\right)
$$

for any function $f \in C^{k}\left(\mathbb{R}^{n}\right)$ such that

$$
f^{* *}(\infty)=(\nabla f)^{* *}(\infty)=\ldots=\left(D^{k-1} f\right)^{* *}(\infty)=0 .
$$

Proof. Like the proof of Theorem 4.1, we take a function $g(t)=$ $t^{-k / n}\left(f^{* *}(t)-f^{*}(t)\right)$ and by inequality $\left\|S_{k} g\right\|_{B} \leq c\|g\|_{A}$ again obtain that

$$
\left\|f^{* *}(t)\right\|_{B} \leq c\left\|t^{-k / n}\left(f^{* *}(t)-f^{*}(t)\right)\right\|_{A}+\|f\|_{L_{1}}\left\|\chi_{(0,|\Omega|)}\right\|_{B},
$$

so that it remains only to refer to the inequality (3.7).

Of course, the term $\|f\|_{L_{1}}$ in the right-hand side of (4.8) may be omitted if $|\Omega|=\infty$ or if $|\Omega|<\infty$ but $B$ is a Banach function space no larger than $L_{1}$. In both these cases we obtain that the space $B=S_{k}\left(A^{\star}\right)$ is the smallest function space giving inequality $\left\|f^{* *}\right\|_{B} \leq c\left\|\left(D^{k} f\right)^{* *}\right\|_{A}$. Unfortunately, analytical description of the space $S_{k}\left(A^{\star}\right)$ may be very difficult and, generally speaking, we can use the result of Theorem 4.2 only for checking that a given space $B$ is suitable for desired Sobolev type embeddings and for estimating its proximity to the optimal space.

\section{Results for ultrasymmetric spaces}

The u.s. spaces are a partial case of r.i. spaces, thus all results of the preceding section are valid for them. However the presentation of results thus obtained is not always the best possible. Sometimes it is better to consider first the function space $\widetilde{E}(\varphi)$ with the norm $\|g\|_{\widetilde{E}(\varphi)}=$ $\|\varphi(t) g(t)\|_{\widetilde{E}}$, to write needed inequalities for it and only then to substitute $g^{*}$ in place of $g$. This way is particularly useful when the corresponding function $g$ is not decreasing.

Theorem 5.1. Let $A$ be an u.s. space with the fundamental function $\varphi=\varphi_{A}(t)$ such that $\rho_{\varphi}<1$ and $\pi_{\varphi}>k / n$. Let $X$ be also a u.s. space with the fundamental function $\varphi_{X}(t)=t^{-k / n} \varphi(t)$ and with the same parameterspace as for $A$. Then $\|f\|_{X} \leq c\left\|\left|D^{k} f\right|\right\|_{A}$ for any $f \in C_{0}^{k}(\Omega)$ and $X$ is the best possible r.i. space for such an inequality. 
Proof. By definition, the norm in the space $A$ is equivalent to $\left\|\varphi(t) f^{*}(t)\right\|_{\widetilde{E}}$. As shown in [27], the Boyd indices of this space are $\pi_{A}=\pi_{\varphi}$ and $\rho_{A}=\rho_{\varphi}$. Thus the space $A$ has both $(P)$ and $Q(k)$ properties, which allows us to apply Corollary of Theorem 4.1, defining the best possible r.i. space $X$ by the norm

$$
\|f\|_{X}=\left\|t^{-k / n} f^{* *}(t)\right\|_{A}=\left\|\varphi(t)\left(t^{-k / n} f^{* *}(t)\right)^{*}\right\|_{\widetilde{E}}=\left\|\varphi(t) t^{-k / n} f^{* *}(t)\right\|_{\widetilde{E}},
$$

since $t^{-k / n} f^{* *}(t)$ is a decreasing function. But $\rho_{X}=\rho_{\varphi}-k / n<1$, hence the norm $\|f\|_{X}$ is equivalent to $\left\|\varphi(t) t^{-k / n} f^{*}(t)\right\|_{\widetilde{E}}$ and we are done.

Now we return to the main assertion given in Theorem 3.1 and study its form for the u.s. spaces.

Theorem 5.2. Let $A$ be a u.s. space with the fundamental function $\varphi=\varphi_{A}(t)$ such that $\pi_{\varphi}>(k-1) / n$ for some positive integer $k \leq n-1$ and with a parameter-space $E$. Then

$$
\left\|\varphi(t) t^{-k / n}\left(f^{* *}(t)-f^{*}(t)\right)\right\|_{\widetilde{E}} \leq c\left\|\left(D^{k} f\right)^{* *}(t)\right\|_{A}
$$

for any function $f \in C^{k}\left(\mathbb{R}^{n}\right)$ such that

$$
f^{* *}(\infty)=(\nabla f)^{* *}(\infty)=\ldots=\left(D^{k-1} f\right)^{* *}(\infty)=0 .
$$

Proof. In contrast to the previous proof, we cannot apply (3.7) directly to $A$, since the function $t^{-k / n}\left(f^{* *}(t)-f^{*}(t)\right)$ may be not decreasing. Let us show that (3.7) holds with the space $\widetilde{E}(\varphi)$ instead of $A$. It is enough to check that $\widetilde{E}(\varphi)$ has $Q(k-1)$ property. We have

$$
\|f(t / s)\|_{\widetilde{L}_{1}(\varphi)}=\int_{0}^{\infty} \varphi(t)|f(t / s)| \frac{d t}{t}=\int_{0}^{\infty} \varphi(t s)|f(t)| \frac{d t}{t} \leq m_{\varphi}(s)\|f(t)\|_{\widetilde{L}_{1}(\varphi)}
$$

and thus $d_{\widetilde{L}_{1}(\varphi)}(s) \leq m_{\varphi}(s)$. Analogously

$$
\|f(t / s)\|_{\widetilde{L}_{\infty}(\varphi)}=\sup _{t} \varphi(t s)|f(t)| \leq m_{\varphi}(s)\|f(t)\|_{\widetilde{L}_{\infty}(\varphi)},
$$

giving $d_{\widetilde{L}_{\infty}(\varphi)}(s) \leq m_{\varphi}(s)$. But $\mathcal{F}\left(\widetilde{L}_{1}(\varphi), \widetilde{L}_{\infty}(\varphi)\right)=\mathcal{F}\left(\widetilde{L}_{1}, \widetilde{L}_{\infty}\right)(\varphi)$, hence the space $\widetilde{E}(\varphi)$ is interpolation for the couple $\left(\widetilde{L}_{1}(\varphi), \widetilde{L}_{\infty}(\varphi)\right)$ and thus $d_{\widetilde{E}(\varphi)}(s) \leq m_{\varphi}(s)$ too. Therefore

$Q(k-1, \widetilde{E}(\varphi))=\int_{1}^{\infty} s^{(k-1) / n} d_{\widetilde{E}(\varphi)}\left(\frac{1}{s}\right) \frac{d s}{s} \leq \int_{1}^{\infty} s^{(k-1) / n} m_{\varphi}\left(\frac{1}{s}\right) \frac{d s}{s}<\infty$,

since $\pi_{\varphi}>(k-1) / n$. 
Now we may apply $(3.7)$ to the space $\widetilde{E}(\varphi)$ that gives

$$
\begin{aligned}
\left\|t^{-k / n}\left(f^{* *}(t)-f^{*}(t)\right)\right\|_{\widetilde{E}(\varphi)} & =\left\|\varphi(t) t^{-k / n}\left(f^{* *}(t)-f^{*}(t)\right)\right\|_{\widetilde{E}} \\
& \leq c\left\|\left(D^{k} f\right)^{* *}(t)\right\|_{\widetilde{E}(\varphi)}=c\left\|\left(D^{k} f\right)^{* *}(t)\right\|_{A},
\end{aligned}
$$

and we are done.

Let us give some applications of the last theorem to particular u.s. spaces.

Example 1. We will use the spaces $L(\infty, p)$, introduced in [2] as sets of functions $f: \Omega \mapsto \mathbb{R}$ such that

$$
\|f\|_{L(\infty, p)}=\left\{\int_{0}^{|\Omega|}\left(f^{* *}(t)-f^{*}(t)\right)^{p} \frac{d t}{t}\right\}^{1 / p}<\infty .
$$

Taking $p=n / k$, we obtain that $\|f\|_{L(\infty, n / k)} \leq c\left\|\left|D^{k} f\right|\right\|_{L_{n / k}}$ for any $f \in C_{0}^{k}(\Omega)$. This result was obtained in [2] for $k=1$ and in [20] for the general case.

Example 2. Let us denote by $\Lambda_{p}$ and $M_{p}$ the Lorentz and the Marcinkiewicz spaces (2.1) with fundamental function $\varphi(t)=t^{1 / p}$. The parameter-space for $\Lambda_{p}$ is $E=L_{1}$, thus (5.1) gives

$$
\left\|t^{1 / p} t^{-k / n}\left(f^{* *}(t)-f^{*}(t)\right)\right\|_{\widetilde{L}_{1}} \leq c\left\|\left(D^{k} f\right)^{* *}(t)\right\|_{\Lambda_{p}}
$$

and for $p=n / k$, we obtain that

$$
\left\|f^{* *}(t)-f^{*}(t)\right\|_{\widetilde{L}_{1}} \leq c\left\|\left|D^{k} f\right|\right\|_{\Lambda_{n / k}}, \quad f \in C_{0}^{k}(\Omega) .
$$

But the left-hand term

$$
\begin{aligned}
\left\|f^{* *}(t)-f^{*}(t)\right\|_{\widetilde{L}_{1}} & =\int_{0}^{|\Omega|}\left(f^{* *}(t)-f^{*}(t)\right) \frac{d t}{t} \\
& =\int_{0}^{|\Omega|}\left(-f^{* *}(t)\right)^{\prime} d t=f^{* *}(0)-f^{* *}(|\Omega|)
\end{aligned}
$$

is equivalent to the norm of $f$ in $L_{\infty}$. Thus we obtain that any function $f$ from $C_{0}^{k}(\Omega)$ satisfies inequality $\|f\|_{L_{\infty}} \leq c\left\|\left|D^{k} f\right|\right\|_{\Lambda_{n / k}}$ (for $k=1$ this inequality was proved in $[6])$.

Example 3. The parameter-space for $M_{p}$ is $E=L_{\infty}$, thus, taking $p=n / k$, we obtain that

$$
\left\|f^{* *}(t)-f^{*}(t)\right\|_{\widetilde{L}_{\infty}} \leq c\left\|\left|D^{k} f\right|\right\|_{M_{n / k}}, \quad f \in C_{0}^{k}(\Omega) .
$$


The set of function $f$ with $\sup _{t}\left|f^{* *}(t)-f^{*}(t)\right|<\infty$ was introduced in [3] under name weak- $L^{\infty}$ and shown to be the r.i. hull of the space $B M O$. Using this terminology, we may say that the set of functions $f \in C_{0}^{k}(\Omega)$ with $\left|D^{k} f\right| \in M_{n / k}$ is contained in weak- $L^{\infty}$.

Except for Example 2, in the case of $\pi_{\varphi} \leq k / n$ all studied till now sets $Y_{k}(A)$, defined by (4.7), turn out to be not linear spaces (see, e.g., [20], Section 4.1); it is very likely that Example 2 is really unique. Thus these sets cannot be taken as optimal r.i. spaces for Sobolev type inequalities. The following theorem defines some "almost" optimal spaces for such a situation.

Theorem 5.3. Let $A=L_{\varphi, E}$ with $\rho_{\varphi}<1$ and let the function $\omega(t)=\varphi(t) t^{-k / n}$ satisfy inequalities $\omega^{\prime}(t)>0$ and

$$
\int_{t}^{|\Omega|} \frac{d s}{s \omega(s)} \leq \frac{c}{t \omega^{\prime}(t)} .
$$

Then $\|f\|_{B} \leq c_{1}\left\|\left|D^{k} f\right|\right\|_{A}$ for any $f \in C_{0}^{k}(\Omega)$, where the u.s. space $B$ is $L_{\psi, E}$ with the parameter-function $\psi(t)=t \omega^{\prime}(t)$.

Proof. We see that $\pi_{\varphi} \geq k / n$, thus the space $A$ satisfies all conditions of Theorem 3.1. Let us show that the space $B$ satisfies the conditions of Theorem 4.2, i.e., that $S_{k}: A \rightarrow B$. As for any positive operator, it suffices to study $S_{k} f$ only for nonnegative functions $f \in A$ so that $\left(S_{k} f\right)^{*}=S_{k} f$. Thus the desired property of $S_{k}$ is equivalent to the inequality

$$
\left\|\psi(t) S_{k} f(t)\right\|_{\widetilde{E}} \leq c\|f\|_{L_{\varphi, E}}, \quad \forall f(t) \geq 0 .
$$

Consider first the case $E=L_{1}$. We have

$$
\begin{aligned}
\left\|\psi(t) S_{k} f(t)\right\|_{\widetilde{L}_{1}} & =\int_{0}^{|\Omega|} \psi(t)\left(\int_{t}^{|\Omega|} s^{k / n} f(s) \frac{d s}{s}\right) \frac{d t}{t} \\
& =\int_{0}^{|\Omega|} \frac{\varphi(s) f(s)}{\omega(s)}\left(\int_{0}^{s} \omega^{\prime}(t) d t\right) \frac{d s}{s} \leq\|\varphi(s) f(s)\|_{\widetilde{L}_{1}} .
\end{aligned}
$$

But the function $\varphi(s) / s$ is decreasing and equivalent to $\varphi^{\prime}(s)$, thus

$$
\|\varphi(s) f(s)\|_{\widetilde{L}_{1}}=\int_{0}^{|\Omega|} \frac{\varphi(s)}{s} f(s) d s \leq \int_{0}^{|\Omega|} \frac{\varphi(s)}{s} f^{*}(s) d s \approx\|f\|_{\Lambda_{\varphi}} .
$$

An analogous estimate for the case $E=L_{\infty}$ can be obtained immediately only for decreasing functions:

$$
\left\|\psi(t) S_{k}\left(f^{*}\right)(t)\right\|_{\widetilde{L}_{\infty}}=\sup _{t} t \omega^{\prime}(t) \int_{t}^{|\Omega|} \frac{\varphi(s) f^{*}(s)}{s \omega(s)} d s
$$




$$
\leq \sup _{t} t \omega^{\prime}(t) \int_{t}^{|\Omega|} \frac{d s}{s \omega(s)}\left\|\varphi(s) f^{*}(s)\right\|_{\widetilde{L}_{\infty}} \leq c\|f\|_{M_{\varphi}}
$$

In order to estimate $S_{k} f$ for arbitrary $f$, let us consider another operator

$$
\left(T_{k} f\right)(t)=t^{k / n-1} \int_{0}^{t} f(s) d s .
$$

As follows from definition, the function $\omega(t) / t$ is decreasing, thus $t \omega^{\prime}(t) \leq$ $\omega(t)$. Therefore

$$
\left\|\psi(t) T_{k}\left(f^{*}\right)(t)\right\|_{\widetilde{L}_{\infty}}=\sup _{t} t^{k / n} \omega^{\prime}(t) \int_{0}^{t} f^{*}(s) d s \leq \sup _{t} \frac{\varphi(t)}{t} \int_{0}^{t} f^{*}(s) d s .
$$

But the last expression is equivalent to $\|f\|_{M_{\varphi}}$, so that we get

$$
\left\|\psi(t)\left(T_{k}+S_{k}\right)\left(f^{*}\right)(t)\right\|_{\widetilde{L}_{\infty}} \leq c\|f\|_{M_{\varphi}} .
$$

On the other hand,

$$
\left(T_{k}+S_{k}\right) f(t)=\int_{0}^{|\Omega|} f(s) \min \left\{t^{k / n-1}, s^{k / n-1}\right\} d s \leq\left(T_{k}+S_{k}\right)\left(f^{*}\right)(t),
$$

since the function $\min \left\{t^{k / n-1}, s^{k / n-1}\right\}$ is decreasing. Thus

$$
\begin{aligned}
\left\|\psi(t) S_{k} f(t)\right\|_{\widetilde{L}_{\infty}} & \leq\left\|\psi(t)\left(T_{k}+S_{k}\right) f(t)\right\|_{\widetilde{L}_{\infty}} \\
& \leq\left\|\psi(t)\left(T_{k}+S_{k}\right)\left(f^{*}\right)(t)\right\|_{\widetilde{L}_{\infty}} \\
& \leq c\|f\|_{M_{\varphi}} .
\end{aligned}
$$

The relations obtained mean that the operator $S_{k}$ is bounded from $\Lambda_{\varphi}$ to $\widetilde{L}_{1}(\psi)$ and from $M_{\varphi}$ to $\widetilde{L}_{\infty}(\psi)$. But, as shown in [27], the triple $\left(\Lambda_{\varphi}, M_{\varphi}, L_{\varphi, E}\right)$ is always interpolation with respect to the triple $\left(\widetilde{L}_{1}(\psi), \widetilde{L}_{\infty}(\psi), \widetilde{E}(\psi)\right)$, hence this operator is also bounded from $L_{\varphi, E}$ to $\widetilde{E}(\psi)$ and the inequality (5.3) is proved.

Sharpness of this theorem can be seen on estimation of functions $\psi(t) S_{k} f(t)$ in $\widetilde{L}_{1}$ and in $\widetilde{L}_{\infty}$. And in some special cases of $\omega(t)$ the space $L_{\psi, E}$ turns out to be optimal among all r.i. spaces satisfying the corresponding Sobolev inequalities. For example, if $\pi_{\omega}>0$ then the function $t \omega^{\prime}(t)$ is equivalent to $\omega(t)$ so that $\psi(t)$ is equivalent to $t^{-k / n} \varphi(t)$. In result we obtain that the space $L_{\psi, E}$ from the last theorem is the same as $X$ from Theorem 5.1. Another example can be given as follows.

Example 4. Let, for simplicity, $|\Omega|=1$ and let $\omega(t)=W\left(\left(\ln \frac{e}{t}\right)^{-1}\right)$, where the function $W(u), 0<u<1$, is of power growth, i.e., there exist 
positive numbers $\alpha, \beta$ such that $\alpha \leq u W^{\prime}(u) / W(u) \leq \beta$. This condition, for instance, is fulfilled if, for sufficiently small $t$,

$$
\omega(t) \approx\left(\ln \frac{e}{t}\right)^{\gamma_{1}}\left(\ln \ln \frac{e}{t}\right)^{\gamma_{2}} \cdots\left(\ln \ldots \ln \frac{e}{t}\right)^{\gamma_{m}}
$$

with arbitrary real numbers $\gamma_{1}, \gamma_{2}, \ldots, \gamma_{m}$ provided $\gamma_{1}<0$. Let us show that the function $\omega(t)=W\left(\left(\ln \frac{e}{t}\right)^{-1}\right)$ with $W(u)$ as above satisfies (5.2) and, moreover, the space $L_{\psi, E}$ from Theorem 5.3 is in this case the smallest among all r.i. spaces $B$ satisfying inequality $\|f\|_{B} \leq c\left\|\left|D^{k} f\right|\right\|_{A}$ for $f \in C_{0}^{k}(\Omega)$.

Indeed, the power growth of $W(u)$ implies that

$$
\frac{t \omega^{\prime}(t)}{\omega(t)}=\frac{W^{\prime}\left(\left(\ln \frac{e}{t}\right)^{-1}\right)\left(\ln \frac{e}{t}\right)^{-2}}{W\left(\left(\ln \frac{e}{t}\right)^{-1}\right)} \approx\left(\ln \frac{e}{t}\right)^{-1} .
$$

Thus

$$
\int_{t}^{|\Omega|} \frac{d s}{s \omega(s)} \leq \frac{1}{\omega(t)} \ln \frac{e}{t} \approx \frac{1}{t \omega^{\prime}(t)}
$$

and the inequality (5.2) is fulfilled.

The equivalence of $t \omega^{\prime}(t)$ and $\omega(t)(\ln (e / t))^{-1}$ ensures also that the space $L_{\psi, E}$ remains the same if we take $\psi(t)=\omega(t)(\ln (e / t))^{-1}$. We should prove that the set $S_{k}(A)$ cannot be included into some r.i. space smaller than $L_{\psi, E}$. It is enough for this to show that, for any function $g(t) \in L_{\psi, E}$, there exists a function $f \in A$ such that $S_{k} f(t) \geq g^{*}(t)$. We construct $f$ by a method similar to one used in [8], Section 5, assuming additionally that $\rho_{E}<1$ (this condition is not necessary but simplifies the proof).

As soon as a function $g(t)$ is given, we define, step by step, the functions

$$
\zeta(t)=\sup _{0<s<t} \psi(s) g^{*}(s), \quad \xi(u)=\zeta\left(e^{-u}\right), \quad z(u)=\xi^{* *}(u)
$$

and, at last, $f(t)=z(\ln (1 / t)) / \varphi(t)$. The properties of the function $\psi(t)$ allow us to apply Theorem 4.2 from [26], which gives that the function $\zeta(t)$ belongs to the same r.i. space as the function $\psi(t) g^{*}(t)$, namely, to $\widetilde{E}$. Subsequently the function $f$ belongs to $\widetilde{E}(\varphi)$. Let us show that, in fact, $f \in L_{\varphi, E}=A$.

Note that, for any $b \in(0,1)$, the function $f(t)$ is bounded on the interval $(b, 1)$, thus the function $f_{1}(t)=f(t) \chi_{(b, 1)}(t)$ belongs to any u.s. space, and we have to investigate only the function $f_{0}(t)=f(t) \chi_{(0, b)}(t)$ for some convenient $b$. We will show that the number $b=e^{-n / k}$ is just such, since 
the function $f_{0}(t)$ with this $b$ is decreasing, coinciding with $f_{0}^{*}(t)$. Indeed,

$$
\begin{aligned}
f^{\prime}(t) & =-\frac{1}{t \varphi(t)}\left[\frac{t \varphi^{\prime}(t)}{\varphi(t)} z\left(\ln \frac{1}{t}\right)+z^{\prime}\left(\ln \frac{1}{t}\right)\right] \\
& \leq-\frac{1}{t \varphi(t)}\left[\frac{k}{n} z\left(\ln \frac{1}{t}\right)+z^{\prime}\left(\ln \frac{1}{t}\right)\right],
\end{aligned}
$$

because

$$
\frac{t \varphi^{\prime}(t)}{\varphi(t)}=\frac{k}{n}+\frac{t \omega^{\prime}(t)}{\omega(t)} \geq \frac{k}{n} .
$$

Setting $t=e^{-u}$, we obtain that

$$
\begin{aligned}
\frac{k}{n} z\left(\ln \frac{1}{t}\right)+z^{\prime}\left(\ln \frac{1}{t}\right) & =\frac{k}{n} z(u)-\frac{1}{u}\left(\xi^{* *}(u)-\xi^{*}(u)\right) \\
& =\left(\frac{k}{n}-\frac{1}{u}\right) z(u)+\frac{1}{u} \xi(u) \geq 0
\end{aligned}
$$

for each $u \geq n / k$, i.e., $f_{0}^{\prime}(t) \leq 0$ for each $t \leq e^{-n / k}=b$ as desired.

Now we can write that

$$
\begin{aligned}
\psi(t) S_{k} f(t) & =\psi(t) \int_{t}^{1} s^{k / n} f(s) \frac{d s}{s} \\
& =\psi(t) \int_{t}^{1} z\left(\ln \frac{1}{s}\right) \frac{d s}{s \omega(s)} \\
& \geq z\left(\ln \frac{1}{t}\right) \psi(t) \int_{t}^{1} \frac{d s}{s \omega(s)}
\end{aligned}
$$

The properties of functions $\omega(t)$ and $W(u)$ provide that $\omega\left(t^{2}\right) \approx \omega(t)$, thus

$$
\int_{t}^{1} \frac{d s}{s \omega(s)} \geq c \int_{t}^{\sqrt{t}} \frac{d s}{s \omega\left(s^{2}\right)} \geq \frac{c}{\omega(t)} \ln \frac{e}{t}=\frac{c}{\psi(t)} .
$$

Therefore

$$
\psi(t) S_{k} f(t) \geq c z\left(\ln \frac{1}{t}\right) \geq c \zeta(t) \geq c \psi(t) g^{*}(t)
$$

and we are done.

Remark. Theorem 5.3 can be also applied to the cases when $\omega^{\prime}(t)=0$ for some $t$, since we always may replace $\omega(t)$ by equivalent strictly increasing functions. For example, we get a new proof for the following result from [7]: $\quad$ if $A=L_{\varphi, E}$ with $\varphi(t)=t^{k / n}, \rho_{E}<1$ and $B=L_{\psi, E}$ with $\psi(t)=(\ln (e / t))^{-1}$, then $\|f\|_{B} \leq c\left\|\left|D^{k} f\right|\right\|_{A}$ for any $f \in C_{0}^{k}(\Omega)$ and the space $L_{\psi, E}$ is optimal among all possible r.i. spaces in this inequality. 


\section{Sobolev type inequalities for Orlicz spaces}

In this section we consider only sets $\Omega$ with finite measure. Recall that a function $\Phi:(0, \infty) \mapsto(0, \infty)$ is said to be a Young function, if it is convex, increasing and

$$
\lim _{t \rightarrow 0+} \frac{\Phi(t)}{t}=\lim _{t \rightarrow \infty} \frac{t}{\Phi(t)}=0 .
$$

Any of such functions defines the corresponding Orlicz space $L_{\Phi}$ as the collection of all measurable functions $f: \Omega \mapsto \mathbb{R}$, for which there exists a positive number $\lambda$ such that

$$
\int_{\Omega} \Phi\left(\frac{|f(x)|}{\lambda}\right) d x<\infty .
$$

This space is usually endowed with the so-called Luxemburg norm

$$
\|f\|_{L_{\Phi}}=\inf \left\{\lambda>0: \int_{\Omega} \Phi\left(\frac{|f(x)|}{\lambda}\right) d x \leq 1\right\} .
$$

The space $L_{\Phi}$ is rearrangement-invariant with a fundamental function $\varphi(t) \approx 1 / \Phi^{-1}(1 / t)$, i.e., up to equivalence of norms, any Orlicz space is uniquely defined by its fundamental function which may be taken as a parameter defining $L_{\Phi}$. Moreover, we can give an explicit formula for the norm in $L_{\Phi}$, expressed via $\varphi$ (see, e.g., [25]):

$$
\|f\|_{L_{\Phi}} \approx \sup _{\|g\|_{L_{1}} \leq 1} \int_{0}^{|\Omega|} f^{*}(t) g^{*}(t) \varphi\left(\frac{1}{g^{*}(t)}\right) d t .
$$

Note that the Boyd indices of the space $L_{\Phi}$ are equal to the extension indices of its fundamental function. Thus if $\pi_{\varphi}>k / n$, we may use (4.6), obtaining the best possible Sobolev type inequality for Orlicz spaces

$$
\|f\|_{B}=\left\|t^{-k / n} f^{* *}(t)\right\|_{L_{\Phi}} \leq c\left\|\left|D^{k} f\right|\right\|_{L_{\Phi}}, \quad f \in C_{0}^{k}(\Omega) .
$$

This case in the theory of Orlicz-Sobolev embeddings is usually named "nonlimiting"; the last formula gives the full solution for such a case. The "superlimiting" inequality $\pi_{\varphi}<k / n$ implies immediately that $L_{\Phi} \hookrightarrow \Lambda_{n / k}$, so that $\left\|\left|D^{k} f\right|\right\|_{L_{\Phi}} \geq c\|f\|_{L_{\infty}}$ due to Example 2. Thus the further discussion may be devoted only to the "limiting" case $\pi_{\varphi}=k / n$.

As in Theorem 5.3, we define the function $\omega(t)=\varphi(t) t^{-1 / p}, p=n / k$, and assume that $\omega^{\prime}(t)>0$. We obtain that $\pi_{\omega}=\pi_{\varphi}-1 / p=0$, hence the function $\omega(t)$ increases slower than any power function with positive exponent. It turns out that this condition is "almost sufficient" for the Orlicz space $L_{\Phi}$ with $\Phi(t) \approx 1 / \varphi^{-1}(1 / t)$ to be ultrasymmetric. Namely, as 
shown by Lorentz in [16], the condition

$$
\exists \lambda>0: \quad \int_{0}^{1} \frac{d t}{\omega^{-1}(\lambda \omega(t))}<\infty
$$

is necessary and sufficient for an Orlicz space $L_{\Phi}$ with the fundamental function $\varphi(t)=\omega(t) t^{1 / p}, p>1$, to have an equivalent norm

$$
\|f\|_{L_{\Phi}} \approx\left\|\varphi(t) f^{*}(t)\right\|_{\widetilde{L}_{p}}=\left\|\omega(t) f^{*}(t)\right\|_{L_{p}}
$$

For instance, we obtain all Orlicz spaces generated by the Young functions

$$
\Phi(u) \approx u^{p}(\ln u)^{\gamma_{1}}(\ln \ln u)^{\gamma_{2}} \cdots(\ln \ln \ldots \ln u)^{\gamma_{n}}, \quad u \geq u_{0},
$$

where $\gamma_{1}<0$ and $\gamma_{2}, \ldots \gamma_{n}$ may be arbitrary real numbers; the corresponding function $\omega(t)$ is exactly as in (5.4).

Using results from Example 4 on the whole, we can now obtain optimal Sobolev type inequalities for the corresponding class of Orlicz spaces.

Theorem 6.1. Let $L_{\Phi}$ be an Orlicz space generated by the Young function $\Phi(u)=u^{p} / F(\ln u)$, where $p=n / k$ and the function $F(v)$ is of power growth for $v \geq 1$. Then $\|f\|_{B} \leq c\left\|\left|D^{k} f\right|\right\|_{L_{\Phi}}$ for any $f \in C_{0}^{k}(\Omega)$, where the space $B$ has the norm

$$
\|f\|_{B}=\left\|t^{-1 / p}\left(\ln \frac{e}{t}\right)^{-1}\left[F\left(\ln \frac{e}{t}\right)\right]^{-1 / p} f^{*}(t)\right\|_{L_{p}}
$$

and is the best possible r.i. space in this inequality.

Proof. First of all let us show that the fundamental function $\varphi(t)$ of the space $L_{\Phi}$ satisfies the Lorentz condition (6.3) and thus $L_{\Phi}$ is a u.s. space with the norm (6.4). The power growth of the function $F(v)$ implies that $\ln F(v) \approx \ln v$, hence $\ln \Phi(u) \approx \ln u$ too. Setting $t=\Phi(u)$, we obtain that $t \approx u^{p} / F(\ln t)$ and thus

$$
u=\Phi^{-1}(t) \approx[t F(\ln t)]^{1 / p} \Longrightarrow \varphi(t) \approx 1 / \Phi^{-1}(1 / t) \approx t^{1 / p}\left[F\left(\ln \frac{e}{t}\right)\right]^{-1 / p}
$$

We obtain that $\omega(t) \approx\left[F\left(\ln \frac{e}{t}\right)\right]^{-1 / p}$, and the condition (6.3) is provided by the power growth of $F(v)$.

Defining the function $W(u)=[F(1 / u)]^{-1 / p}$, we readily obtain that it is of power growth for $u \in(0,1)$, so that the function $\omega(t)$ satisfies all conditions of Example 4. Moreover, the corresponding function $\psi(t)=\omega(t)(\ln (e / t))$ is such that the space $B$ coincides with the u.s. space $L_{\psi, L_{p}}$ and our theorem 
turns out to be a partial case of Example 4 when the parameter space $E=L_{p}$.

Remark. The space $B$ from the last theorem can be represented as a weighted Orlicz space, namely, the definition of its norm (6.5) is identical to $\|f\|_{B}=\left\|t^{-k / n} f(t)\right\|_{L_{\Psi}}$, where the Young function $\Psi(u)=\Phi(u) / \ln ^{p} u$. Thus the considered "limiting" case of Orlicz-Sobolev embeddings becomes similar to "nonlimiting" case (6.2), where we had $L_{\Psi}=L_{\Phi}$.

Example 5. The Lorentz condition (6.3) is fulfilled also for some more rapidly increasing functions $\omega(t)$ such as

$$
\omega(t)=e^{-c \ln ^{\varepsilon}(1 / t)} \quad \text { for } \quad c>0, \quad 0<\varepsilon<1, \quad t \leq t_{0} .
$$

The corresponding Young function $\Phi(u)$ can be shown to satisfy inequality

$$
u^{p} e^{-c_{1} \ln ^{\varepsilon} u} \leq \Phi(u) \leq u^{p} e^{-c_{2} \ln ^{\varepsilon} u}, \quad u \geq u_{0},
$$

with some positive constants $c_{1}, c_{2}$ depending on $\varepsilon$ and $p$. The function (6.6) does not satisfy the conditions of Example 4, but the inequality (5.2) is valid for it. Indeed, the change of variable $v=(\ln (1 / s))^{\varepsilon}$ gives

$$
\int_{t}^{1} \frac{d s}{s \omega(s)}=r \int_{1}^{z} v^{r-1} e^{c v} d v, \quad \text { where } z=\left(\ln \frac{1}{t}\right)^{\varepsilon}, r=\frac{1}{\varepsilon}
$$

and then integration by parts enables us to separate the principal part of this integral (for $z \rightarrow \infty$ ) and to get an estimate

$$
\int_{t}^{1} \frac{d s}{s \omega(s)} \leq C z^{r-1} e^{c z}=C\left(\ln \frac{1}{t}\right)^{1-\varepsilon} e^{c \ln ^{\varepsilon}(1 / t)} \approx \frac{1}{t \omega^{\prime}(t)},
$$

as desired.

Applying Theorem 5.3, we obtain that $\|f\|_{B} \leq c\left\|\left|D^{k} f\right|\right\|_{L_{\Phi}}$ for $f \in$ $C_{0}^{k}(\Omega)$, where $B=L_{\psi, L_{p}}$ with

$$
\psi(t)=t \omega^{\prime}(t)=e^{-c \ln ^{\varepsilon}(1 / t)}[\ln (1 / t)]^{\varepsilon-1} .
$$

This means that $B$ again is a weighted Orlicz space $L_{\Psi}\left(t^{-k / n}\right)$ generated now by the Young function $\Psi(u)=\Phi(u)(\ln u)^{p(\varepsilon-1)}$.

If one defines a function $\omega(t)$ from (6.6) with $\varepsilon=1$, this function becomes a power function, corresponding to the "nonlimiting" case of Orlicz-Sobolev embeddings. However there are increasing functions $\omega(t)$ which are intermediate between functions (6.6) with $\varepsilon<1$ and power functions - for example, 


$$
\omega(t)=\exp \left\{-\ln \frac{1}{t} /\left(\ln \ln \frac{1}{t}\right)^{\varepsilon}\right\}=t^{[\ln \ln (1 / t)]^{-\varepsilon}}, \quad \varepsilon>0, \quad t \leq t_{0} .
$$

Unfortunately, this function does not satisfy the Lorentz condition (6.3) and the corresponding Orlicz space $L_{\Phi}$ is not a u.s. space. Nevertheless, a rather sharp inequality for this case of Orlicz-Sobolev embeddings can be obtained by the following statement.

Theorem 6.2. Let an Orlicz space $L_{\Phi}$ have a fundamental function $\varphi(t)=t^{1 / p} \omega(t)$ where $\omega(t)$ is an increasing function with zero extension indices. Let the operator (4.5) is bounded from u.s. space $L_{\varphi, L_{p}}$ to u.s. space $B=L_{\psi, L_{p}}$. Then $\|f\|_{B} \leq c\left\|\left|D^{k} f\right|\right\|_{L_{\Phi}}$ for all $f \in C_{0}^{k}(\Omega)$.

Proof. Due to Theorem 4.2 it suffices to show that $L_{\Phi} \hookrightarrow L_{\varphi, L_{p}}$. We will use the formula (6.1) for the norm in $L_{\Phi}$. For each function $g(t)$ such that $\|g\|_{L_{1}} \leq 1$ and for any $t \leq|\Omega|$, we have that $t g^{*}(t) \leq \int_{0}^{t} g^{*}(s) d s \leq 1$, so that $g^{*}(t) \leq 1 / t$. Therefore

$$
\begin{aligned}
\|f\|_{L_{\Phi}} & =\sup _{\|g\|_{L_{1}} \leq 1} \int_{0}^{|\Omega|} f^{*}(t)\left(g^{*}(t)\right)^{1-1 / p} \omega\left(1 / g^{*}(t)\right) d t \\
& \geq \sup _{\|g\|_{L_{1}} \leq 1} \int_{0}^{|\Omega|} f^{*}(t)\left(g^{*}(t)\right)^{1-1 / p} \omega(t) d t .
\end{aligned}
$$

Setting $h^{*}(t)=\left(g^{*}(t)\right)^{1-1 / p}$, we obtain that $\|g\|_{L_{1}} \leq 1$ if and only if $\|h\|_{L_{q}} \leq 1$ for $1 / q=1-1 / p$ and thus

$$
\|f\|_{L_{\Phi}} \geq \sup _{\|h\|_{L_{q}} \leq 1} \int_{0}^{|\Omega|} f^{*}(t) h^{*}(t) \omega(t) d t .
$$

Consider now a quasilinear operator

$$
U f(t)=(U f)^{*}(t)=\frac{1}{\omega(t)} \sup _{s>t} \omega(s) f^{*}(s)
$$

for which one gets immediately that

$$
\|U f\|_{M_{\omega}}=\sup _{t} \omega(t)(U f)^{*}(t)=\sup _{t} \sup _{s>t} \omega(s) f^{*}(s)=\|f\|_{M_{\omega}} .
$$

Let $\xi(t)=t^{1 / r} \omega(t)$ with some $r<p$. Then, analogously,

$$
\|U f\|_{M_{\xi}}=\sup _{t} t^{1 / r} \sup _{s>t} \omega(s) f^{*}(s) \leq \sup _{t} \sup _{s>t} s^{1 / r} \omega(s) f^{*}(s)=\|f\|_{M_{\xi}},
$$


hence the operator $U$ is bounded in both spaces $M_{\omega}$ and $M_{\xi}$. At the same time $\rho_{\omega}<\pi_{\varphi}, \rho_{\varphi}<\pi_{\xi}$, since $\rho_{\omega}=0, \pi_{\varphi}=\rho_{\varphi}=1 / p$ and $\pi_{\xi}=1 / r$. This implies that the space $L_{\Phi}$ is a real interpolation one between $M_{\omega}$ and $M_{\xi}$ (see, e.g., [14]) and thus the operator $U$ is bounded in $L_{\Phi}$ as well. Applying inequality (6.8), we obtain that

$\|f\|_{L_{\Phi}} \geq c\|U f\|_{L_{\Phi}} \geq c \sup _{\|h\|_{L_{q}} \leq 1} \int_{0}^{|\Omega|} U f(t) h^{*}(t) \omega(t) d t=c\|U f(t) \omega(t)\|_{L_{p}}$,

because the function $U f(t) \omega(t)$ is decreasing. But $U f(t) \geq f^{*}(t)$, whence

$$
\|f\|_{L_{\Phi}} \geq c\left\|f^{*}(t) \omega(t)\right\|_{L_{p}}=c\left\|\varphi(t) f^{*}(t)\right\|_{\widetilde{L}_{p}}
$$

as desired.

Example 6. Let us get a Sobolev type inequality for Orlicz space $L_{\Phi}$ with the fundamental function $\varphi(t)=t^{k / n} \omega(t)$ when the function $\omega(t)$ is defined by (6.7). By the last theorem it suffices to find (as sharp as possible) a function $\psi(t)$ such that $S_{k}: L_{\varphi, L_{p}} \rightarrow L_{\psi, L_{p}}$. The only tool we have for this is the proof of Theorem 5.3, requiring to show that the function (6.7) satisfies inequality (5.2). Unfortunately, the direct computation of the corresponding integral (as in Example 5) is now very complicated, so we will use another way for the proof.

Consider an auxiliary function

$$
\delta(u)=e^{u / \ln ^{\varepsilon} u} \ln ^{\varepsilon} u=\ln ^{\varepsilon} u / \omega\left(e^{-u}\right), \quad u \geq u_{0} .
$$

Then

$$
\delta^{\prime}(u)=e^{u / \ln ^{\varepsilon} u}\left(1-\frac{\varepsilon}{\ln u}+\frac{\varepsilon \ln ^{\varepsilon-1} u}{u}\right) \approx 1 / \omega\left(e^{-u}\right), \quad u \geq u_{0},
$$

and thus

$$
\int_{t}^{|\Omega|} \frac{d s}{s \omega(s)} \approx \int_{t}^{t_{0}} \delta^{\prime}\left(\ln \frac{1}{s}\right) \frac{d s}{s} \leq \delta\left(\ln \frac{1}{t}\right) .
$$

On the other hand, for $u \geq u_{0}$,

$$
\omega^{\prime}\left(e^{-u}\right) e^{-u}=\frac{\delta^{\prime}(u)}{\delta^{2}(u)} \ln ^{\varepsilon} u-\frac{\varepsilon}{u \delta(u)} \ln ^{\varepsilon-1} u=\frac{1}{\delta(u)}\left(1-\frac{\varepsilon}{\ln u}\right) \approx \frac{1}{\delta(u)},
$$

so that $\delta\left(\ln \frac{1}{t}\right) \approx 1 /\left(t \omega^{\prime}(t)\right)$, and the inequality (5.2) is proved.

Now we may apply Theorem 5.3, computing the function

$$
\psi(t)=t \omega^{\prime}(t) \approx 1 / \delta\left(\ln \frac{1}{t}\right)=\omega(t) /\left(\ln \ln \frac{1}{t}\right)^{\varepsilon}, \quad t \leq t_{0},
$$


and obtaining the inequality $\|f\|_{B} \leq c\left\|\left|D^{k} f\right|\right\|_{L_{\Phi}}$ for all $f \in C_{0}^{k}(\Omega)$, where the space $B=L_{\psi, L_{p}}$ has the (quasi)norm

$$
\|f\|_{B}=\left\|t^{-(k / n)+[\ln \ln (1 / t)]^{-\varepsilon}}\left(\ln \ln \frac{1}{t}\right)^{-\varepsilon} f^{*}(t)\right\|_{L_{n / k}} .
$$

\section{References}

[1] D. R. Adams, A sharp inequality of J. Moser for higher order derivatives, Ann. Math., 128 (1988), 385-398.

[2] J. Bastero, M. Milman and F. Ruiz, A note on $L(\infty, q)$ spaces and Sobolev embeddings, Indiana Univ. Math. J., 52 (2003), 1215-1230.

[3] C. Bennett and R. Sharpley, Interpolation of Operators, Academic Press, New York, 1988.

[4] H. Brézis and S. Wainger, A note on limiting cases of Sobolev embeddings and convolution inequalities, Comm. Partial Diff. Eq., 5 (1980), 773-789.

[5] A. Cianchi, Optimal Orlicz-Sobolev embeddings, Revista Mat. Iberoamer., 20 (2004), 427-474.

[6] A. Cianchi and L. Pick, Sobolev embeddings into BMO, VMO and $L_{\infty}$, Ark. Mat., 36 (1998), 317-340.

[7] M. Cwikel and E. Pustylnik, Sobolev type embeddings in the limiting case, J. Fourier Anal. Appl., 4 (1998), 433-446.

[8] M. Cwikel and E. Pustylnik, Weak type interpolation near "endpoint" spaces, J. Funct. Anal., 171 (2000), 235-277.

[9] V. A. Dikarev, Imbedding theorems for a class of function spaces, Dokl. Akad. Nauk SSSR, 168 (1966), 1239-1241; English transl. in Sov. Math. Dokl., 7 (1966), 805-807.

[10] D. E. Edmunds, R. Kerman and L. Pick, Optimal Sobolev embeddings involving rearrangement invariant quasi-norms, J. Funct. Anal., 170 (2000), 307-355.

[11] J. J. F. Fournier, Mixed norms and rearrangements: Sobolev's inequality and Littlewood's inequality, Ann. Mat. Pura Appl., 148 (1987), 51-76.

[12] K. Hansson, Imbedding theorems of Sobolev type in potential theory, Math. Scand., 45 (1979), 77-102.

[13] V. I. Kolyada, Rearrangements of functions and embedding theorems, Uspekhi Mat. Nauk, 44 (1989), 61-95; English transl. in Russian Math. Surveys, 44 (1989), 73-117. 
[14] S. G. Kre厃ّn, Ju. I. Petunin and E. M. Semenov, Interpolation of linear operators, Transl. of Math. Monographs, 54, Amer. Math. Soc., Providence R.I., 1982.

[15] N. Krugljak, L. Maligranda and L. E. Person, On an elementary approach to the fractional Hardy inequality, Proc. Amer. Math. Soc., 128 (2000), 727-734.

[16] G. G. Lorentz, Relations between function spaces, Proc. Amer. Math. Soc., 12 (1961), 127-132.

[17] J. Malý and L. Pick, An elementary proof of sharp Sobolev embeddings, Proc. Amer. Math. Soc., 130 (2002), 555-563.

[18] V. G. Maz'ya, On certain integral inequalities for functions of many variables, Problems of Math. Analysis, Leningrad State University, 3 (1972), 33-68 (in Russian); English transl. in J. Sov. Math., 1 (1973), 205-234.

[19] V. G. Maz'ya, Sobolev Spaces, Springer-Verlag, New York, 1985.

[20] M. Milman and E. Pustylnik, On sharp higher order Sobolev embeddings, Comm. Contemp. Math., 6 (2004), 495-511.

[21] R. O'Neil, Convolution operators and $L(p, q)$ spaces, Duke Math. J., 30 (1963), 129-142.

[22] J. Peetre, Espaces d'interpolation et théorème de Soboleff, Ann. Inst. Fourier, 16 (1966), 279-317.

[23] L. Pick, Optimal Sobolev embeddings, Rudolf-Lipschitz-Vorlesungs, 43, Bonn, 2002.

[24] S. I. Pokhozhaev, On eigenfunctions of the equation $\Delta u+\lambda f(u)=0$, Dokl. Akad. Nauk SSSR, 165 (1965), 36-39; English transl. in Soviet Math. Dokl., 6 (1965), 1408-1411.

[25] E. Pustylnik, On optimal interpolation and some interpolation properties of Orlicz spaces, Dokl. Akad. Nauk SSSR, 269 (1983), 292295; English transl. in Sov. Math. Dokl., 27 (1983), 333-336.

[26] E. Pustylnik, Optimal interpolation in spaces of Lorentz-Zygmund type, J. d'Analyse Math., 79 (1999), 113-157.

[27] E. Pustylnik, Ultrasymmetric spaces, J. London Math. Soc., 68 (2003), 165-182.

[28] Y. Sagher and P. Shvartsman, Rearrangement-function inequalities and interpolation theory, J. Approx. Theory, 119 (2002), 214-251.

[29] E. M. Stein, Singular integrals and differentiability of functions, 1970, Princeton University Press.

[30] N. S. Trudinger, On imbeddings into Orlicz spaces and some applications, J. Math. Mech., 17 (1967), 473-483. 
[31] P. L. Ul'yanov, The embedding of certain classes $H_{p}^{\omega}$ of functions, Izv. Akad. Nauk SSSR, Ser. Mat., 32 (1968), 649-686; English transl. in Math. USSR-Izv., 2 (1968), 601-637.

[32] V. I. Yudovich, On certain estimates related to integral operators and solutions of elliptic equations, Dokl. Akad. Nauk SSSR, 139 (1961), 805-808 (in Russian); English transl. in Soviet Math. Dokl., 2 (1961), 746-749.

Department of Mathematics

Technion - Israel Institute of Technology

Haifa 32000

Israel

(E-mail : evg@techunix.technion.ac.il)

(Received : December 2003) 


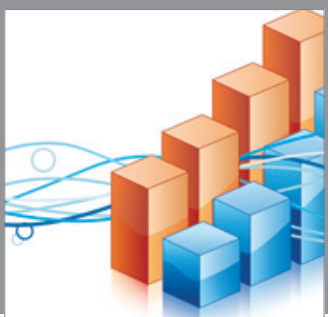

Advances in

Operations Research

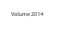

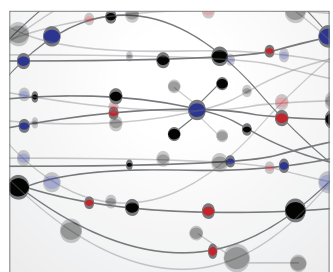

\section{The Scientific} World Journal
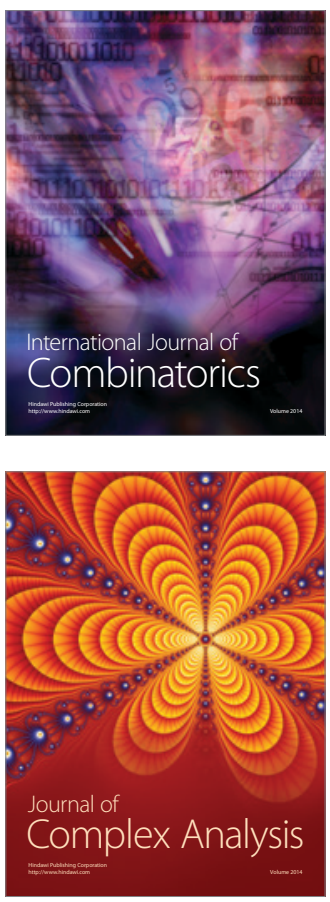

International Journal of

Mathematics and

Mathematical

Sciences
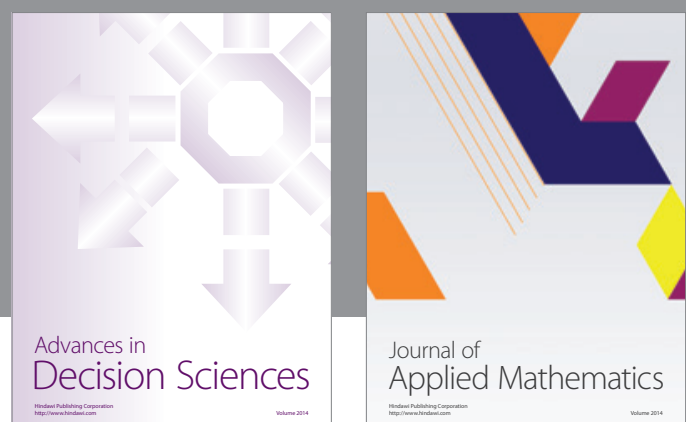

Journal of

Applied Mathematics
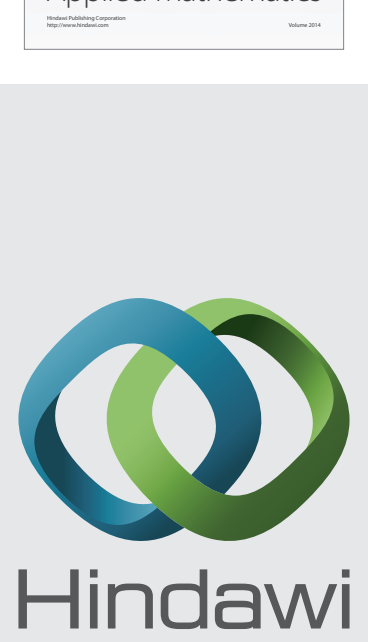

Submit your manuscripts at http://www.hindawi.com
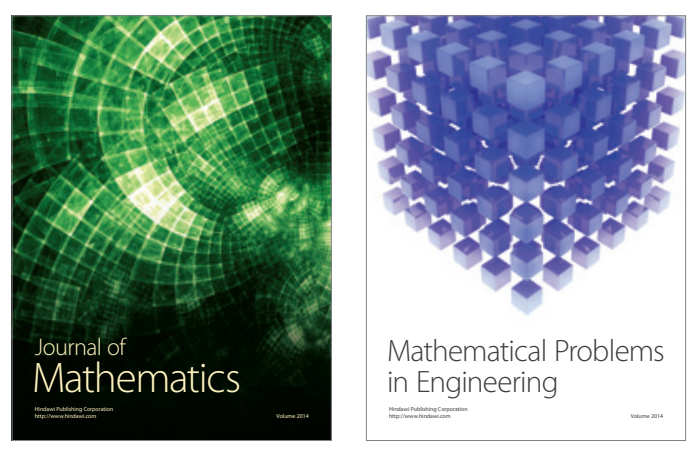

Mathematical Problems in Engineering
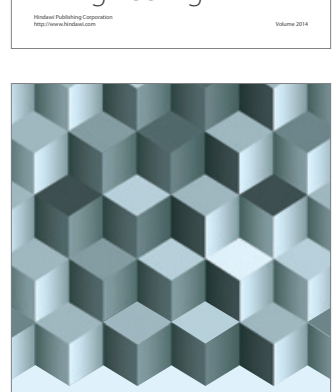

Journal of

Function Spaces
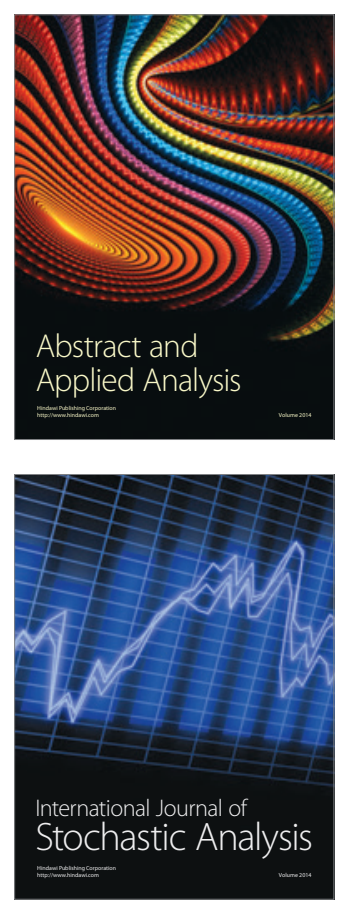

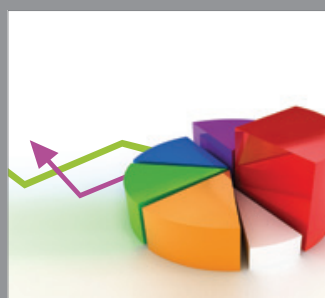

ournal of

Probability and Statistics

Promensencen
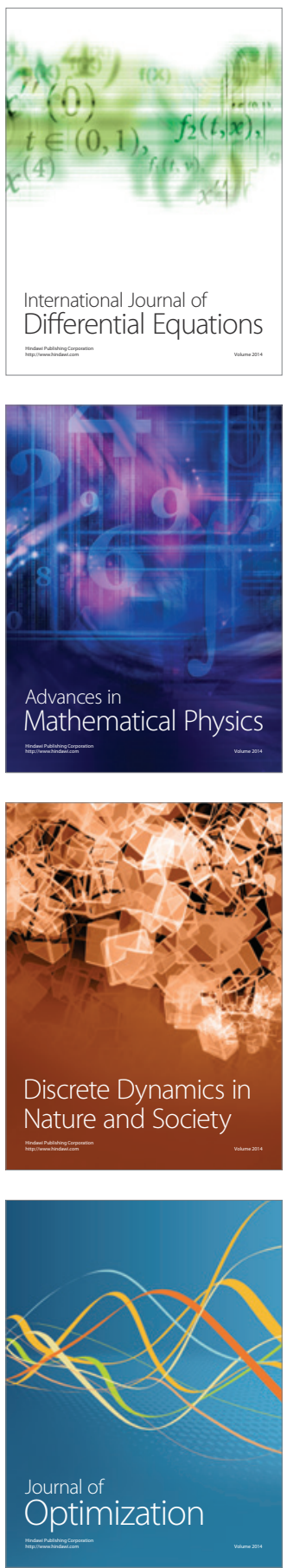